\title{
Vorticity Confinement method applied to flow around an Ahmed body and comparison with experiments
}

\author{
M. Forman ${ }^{1}$, H. Jasak ${ }^{2}$, J. Volavy ${ }^{1}$, M. Jicha ${ }^{1}$, C. Othmer ${ }^{3}$ \\ \& H. P. Bensler ${ }^{3}$ \\ ${ }^{1}$ Brno University of Technology, Faculty of Mechanical Engineering, \\ Energy Institute, Czech Republic \\ ${ }^{2}$ Wikki, Ltd., UK \\ ${ }^{3}$ Volkswagen, Germany
}

\begin{abstract}
In this paper, the Vorticity Confinement method (VC) is presented for the calculation of flow field around a bluff body. The VC method was originally proposed by Fan et al. ("Computing blunt body flows on coarse grids using vorticity confinement", J. Fluids Engineering, 124(4):1-18, Dec 2002) for the computation of thin vortical regions in the high Reynolds number incompressible flows. Steinhoff et al. (Large Eddy Simulation: Computing Turbulent Flow Dynamics, Chapter 12: Turbulent Flow Simulations Using Vorticity Confinement: Cambridge University Press, 2006) claim that VC is capable of capturing most of the main features of high-Re turbulent flows without the massive mesh resolution needed for Reynolds-Averaged Navier-Stokes (RANS) solutions or Large Eddy Simulation (LES). The aim of the method is to capture small-scale vortical structures efficiently. The presented study assesses the VC method (namely the first formulation, referred to as VC-1, which involves first derivatives of velocity), for external aerodynamics presented here on a well documented simplified car - Ahmed bluff body with different slant angles, namely $25^{\circ}$ and $35^{\circ}$. These slant angles were chosen because the $35^{\circ}$ slant angle supports the boundary layer separation and therefore stronger wake (also stronger oscillations of the wake), while at the $25^{\circ}$ slant angle no separation takes part and the wake is much weaker and also more stable. The VC method was applied using different values for the diffusion parameter and confinement
\end{abstract}


parameter, respectively. In addition, different a mesh size was tested. The comparison results are presented for RANS models. The RANS simulations were carried out using the $\mathrm{k}-\omega \mathrm{SST}$ model run in transience with second-order differencing in time and space. OpenFOAM code with wall functions was used. The experimental data used for validation were taken from Lienhart et al. (Flow and Turbulence Structures in the Wake of a Simplified Car Model (Ahmed Model), Measurements MOVA- project, online database: http://cfd.mace. manchester.ac.uk/ercoftac/) from the ERCOFTAC database. Compared are velocity profiles in different sections along the Ahmed body for both $25^{\circ}$ and $35^{\circ}$ slant angles, and forces acting on the body and drag coefficient.

Keywords: turbulent flow, Vorticity Confinement, Ahmed body, experiments.

\section{Introduction}

Many researchers and engineers are constantly looking for a rapid, yet correct method for external aerodynamics in turbulent flow with massive separation and vortexes. It is well known that standard CFD methods are not capable of conserving vortices over a sufficient period of time because of the numerical diffusion that is required to stabilize the numerical scheme. The solution obtained may be strongly dependent on the models and on the discretization used (e.g. turbulence model or grid) as soon as the flow is sufficiently complex with flow separation. Consequently, a large effort is still needed so that the flow physics is correctly represented. A natural way to tackle such a problem is to use high-order schemes and/or automatic grid refinement techniques in order to increase the accuracy of the resolution and thus avoid a too fast dissipation of the vortical structures [5-8]. However, such approaches are expensive and difficult to apply to realistic aerodynamic configurations with the present computer resources.

Until now there has been no universally acceptable turbulence model that can provide uniformly good results for engineering problems with separated flows. Algebraic models are simple to use, but are quite unreliable for separation flows because of the Boussinesq approximation. One-equation models have achieved closer agreement with experimental measurements for a limited number of separated flows than is possible with algebraic models, but the length scale for each new application needs to be specified. The $k-\varepsilon$ model is the most widely used in the family of two equation models; unfortunately, it is inaccurate for flows with adverse pressure gradients, separation and recirculation. It appears that all two-equation models are inaccurate for the flows around surfaces with large curvatures, separations, sudden change in mean strain rate, rotation, vortex shedding, and 3D unsteady motions.

Alternative techniques capable to overcome these difficulties at reasonable cost have been developed. We have to mention an original Steinhoff's Vorticity Confinement method [9]. This technique modifies the incompressible NavierStokes equations by adding a well-chosen source term into the momentum equations, which concentrates vortices over a small number of grid cells and allows one to convect them without diffusion over a long period of time. This 
confinement source term is aimed to be a model of the vortex flow structures. Such methodology has been successfully applied to very different types of flowfields, from simple vortices up to complex rotor-fuselage interactions, using an extension of the method to general body surfaces with simple Cartesian grids and even to massively separated flows. The method has also been generalized to compressible flows considering the corresponding source term as a body force.

\section{Vorticity confinement model}

The vorticity confinement model is derived by adding a pair of terms to the transient incompressible laminar Navier-Stokes system, with the purpose of controlling the diffusive discretisation error and vorticity capturing. The idea of the model is that numerical discretisation methods introduce unacceptable levels of numerical diffusion and fail to resolve essential small-scale features of the flow. This can be clearly seen when a CFD code is used to transport an underresolved vortex over a large distance or one attempts to track a wave along a large distance. Authors [2] claim that $\mathrm{VC}$ is capable of capturing most of the main features of high-Re turbulent flows without the massive mesh resolution needed for Reynolds-Averaged Navier Stokes (RANS) solutions or Large Eddy Simulation (LES). This is achieved by adding a negative eddy-type viscosity which saturates in a stable manner and counteracts the positive viscosity added by inappropriate discretisation.

There exist two formulations of the VC model. The first, referred to as VC-1, involves first derivatives of velocity, whereas the second (VC-2) involves second derivatives [10]. VC-1 was chosen for this study due to its robustness.

Modified Navier-Stokes system including the pair of VC terms reads as follows:

$$
\begin{gathered}
\nabla \cdot \mathbf{u}=0 \\
\frac{\partial \mathbf{u}}{\partial t}+\nabla \cdot(\mathbf{u u})-\nabla \cdot\left(v_{\boldsymbol{v} \boldsymbol{c}} \nabla \mathbf{u}\right)=-\nabla p+\varepsilon \mathrm{s}
\end{gathered}
$$

where $v_{v c}$ is the diffusive coefficient which controls the dissipation of the vorticity part es. The second confinement term is calculated for the curl of velocity, using a user-defined constant $\mathbf{s}$ as follows:

$$
\mathrm{s}=\frac{\nabla|\omega|}{|\nabla| \omega||} \omega
$$

where $\omega$ is curl of velocity defined as $\omega=\nabla \times \mathrm{u}$.

From the presented set of equations, two parameters need to be set. It is the diffusion parameter $v_{v c}$ and confinement parameter $\varepsilon$. The first confinement constant $v_{v c}$ is clearly related to fluid viscosity and numerical diffusion of the base (un-confined) solver. Its dependence of the Re number is clear. However, the second confinement constant $\varepsilon$ is not dimensionless and its scaling with geometry, Re number and possibly other parameters is unknown. A consequence is that confinement tuning will be strictly problem-dependent, but presumably 
constant over a range of related problems. In his paper, Vaughn [12] offers a way of estimating confinement parameters based on an analysis of the confinement parameter and the viscous terms. He suggested two ways how to calculate the parameters giving two different confinement parameters.

By assuming turbulence production to be homogenous and turbulent viscosity to be spatially constant for the local flow, it is possible to express the production in terms of vorticity. Since the laminar and turbulent viscous terms of the Navier-Stokes equations have the same form (due to Boussinesq approximation) the laminar components of the equation can likewise be articulated in terms of vorticity. Furthermore, it is assumed that $v_{v c}$ is a measure of local viscosity, combining its laminar and "turbulent" contribution. By performing an order-ofmagnitude analysis, the first way how to calculate confinement parameter can be written in the form:

$$
\varepsilon=\sqrt{2} \frac{v_{v c}}{u h}
$$

where $h$ is the representative dimension of local cell size (here cell size close to the bluff body) and $u$ is free stream velocity (in our case mean velocity $40 \mathrm{~m} / \mathrm{s}$ ). Second form is defined as:

$$
\varepsilon=\frac{\sqrt{2}}{\operatorname{Re}_{L}} \frac{L}{h}\left(1+0.1344 \sqrt{\operatorname{Re}_{L}}\right)
$$

Both equations assume the diffusion coefficient $v_{v c}$ to consist of viscosity. While first form (4) takes only laminar viscosity into account, the second form (5) assumes the turbulence using turbulent Reynolds number calculated with respect to the global size $L$ of the bluff body.

\section{Model setup}

\subsection{Geometry}

The presented study assesses the VC method for external aerodynamics presented here on a well documented simplified car - Ahmed bluff body [3]. The tunnel in which the Ahmed body was placed has $1.87 \times 1.4 \mathrm{~m}^{2}$ in cross-section and is $6 \mathrm{~m}$ long. The flow parameters are:

kinematic viscosity $15 \times 10^{-6} \mathrm{~m}^{2} / \mathrm{s}$

bulk velocity $U_{b}=40 \mathrm{~m} / \mathrm{s}$

height of the body $h=288 \mathrm{~mm}$

Reynolds number based on height of the bluff body $R e=768000$.

The geometry of the bluff body is depicted in Figure 1.

The experimental data used for validation are from measurements of Lienhart et al. [4] and were downloaded from the ERCOFTAC online database.

\subsection{Computational mesh}

Computational mesh for all cases was created in program STAR-CCM+ using trimmed mesh (unstructured hexahedral mesh with "one to many" connections). 
Such a mesh allows local refinement in the place of interest without affecting the mesh structure further from the place. The base size of the mesh in the free flow further away from the Ahmed body was held constant for all cases at value of $0.1 \mathrm{~m}$. To study the grid dependence of VC method, three grid sizes were used as shown in the Table 1 . The mesh was refined from the base size in the vicinity of the bluff body as well as in the region of recirculation. Only on coarse mesh the front part of the body has mesh size of $2.5 \mathrm{~cm}$ to capture the curvature of the front part of the body as depicted in Figure 2. For the fine and extra fine mesh the surface size is kept constant along the body surface except the stubs where the mesh has the size of approx. $3 \mathrm{~mm}$ (see Figure 3).

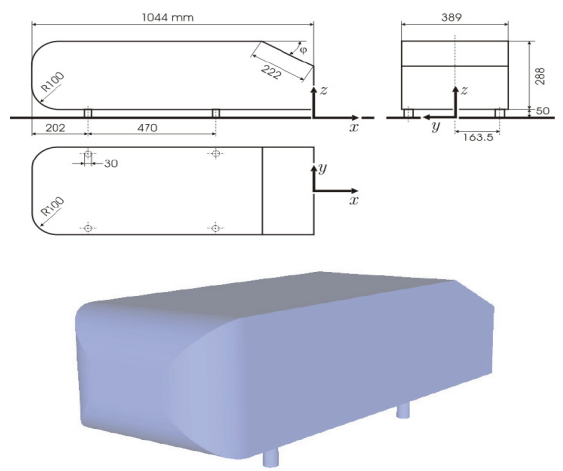

Figure 1: $\quad$ Ahmed body.

Table 1.

\begin{tabular}{|c|c|c|c|}
\hline & $\begin{array}{c}\text { Global } \\
\text { mesh size }\end{array}$ & $\begin{array}{c}\text { Local } \\
\text { mesh size }\end{array}$ & $\begin{array}{c}\text { cell } \\
\text { number }\end{array}$ \\
\hline Coarse & $0.10 \mathrm{~m}$ & $0.0250 \mathrm{~m}$ & 227,046 \\
\hline Fine & $0.10 \mathrm{~m}$ & $0.0125 \mathrm{~m}$ & $1,375,659$ \\
\hline Extra fine & $0.05 \mathrm{~m}$ & $0.0050 \mathrm{~m}$ & $2,186,569$ \\
\hline
\end{tabular}

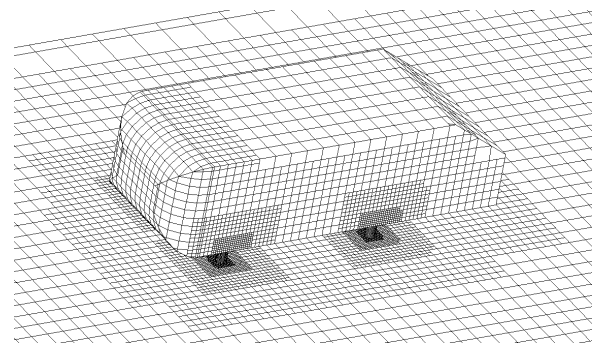

Figure 2: $\quad$ Surface mesh detail with coarse mesh settings. 


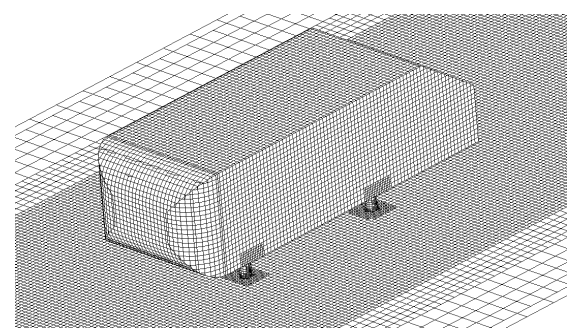

Figure 3: $\quad$ Surface mesh detail with extra fine mesh settings.

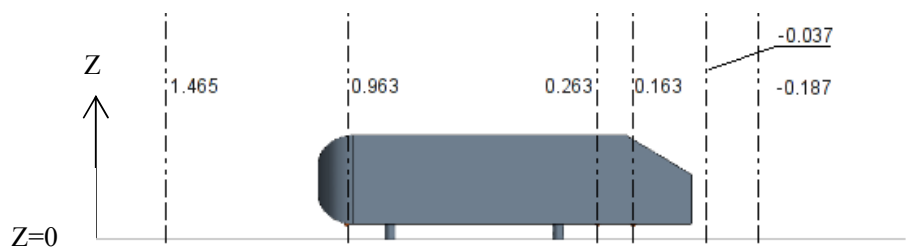

Figure 4: $\quad$ Cross section specification (X positions).

\subsection{Solver settings in OpenFOAM}

The VC solver developed by H. Jasak was used for all computations. The solver is transient by nature therefore all computations have been carried as transient. Hence the results presented in the study are averaged over several seconds to capture 4 and more cycles of vortex shedding behind the bluff body. For the solution version 1.5.x of OpenFOAM, the PISO algorithm was used using GAMG solver with FDIC preconditioner for pressure and PBiCG solver with DILU preconditioner for velocities. The coarse mesh has maximal nonorthogonality of 45 degrees and skewness of 3.5, while the fine mesh has maximal non-orthogonality of 50 degrees and maximum skewness of 2.9. The solver has been run with 2 non-orthogonal correctors.

\section{Results}

Results are presented for two geometries of the Ahmed body, namely for Ahmed body with $25^{\circ}$ and $35^{\circ}$ slant angles, respectively. These two geometries have been chosen for the different flow-field. The $35^{\circ}$ slant angle supports the boundary layer separation and therefore stronger wake (also stronger oscillations of the wake) while at the $25^{\circ}$ slant angle no separation takes part and the wake is much weaker and also more stable. As principle results, velocity profiles will be evaluated in several cross sections along the bluff body and close to slant angle. The locations of the cross sections are marked in Figure 4. 


\subsection{Ahmed body $-25^{\circ}$ slant angle}

Results are presented for fine mesh - for details see Tab.1. In the Figure 5, calculations are presented for velocity profiles with the fine mesh. Velocities are presented in the Z-direction perpendicular to the floor plane, with zero value on the floor. As we can see, the values of velocities are very close to the ones from the measurements (Ercoftac points) and the maximum error is only 3 per cent. However, from the figure there is no visible trend for the improvement with changing of vorticity parameter $\varepsilon$. Courant number $\mathrm{Co}$, which is the measure of the time step, was kept 8 . The results from these computations are encouraging, while the mesh, although having over 1 million of cells has the smallest cells only $1.25 \mathrm{~cm}$ in size. The only noticeable discrepancy can be detected close and after the stagnation point, in the location $X=0.963$. Calculations were also done on the coarse mesh (not shown here), where - unlike for the fine mesh -an influence of different values of vorticity parameter $\varepsilon$ can be seen, this mainly in
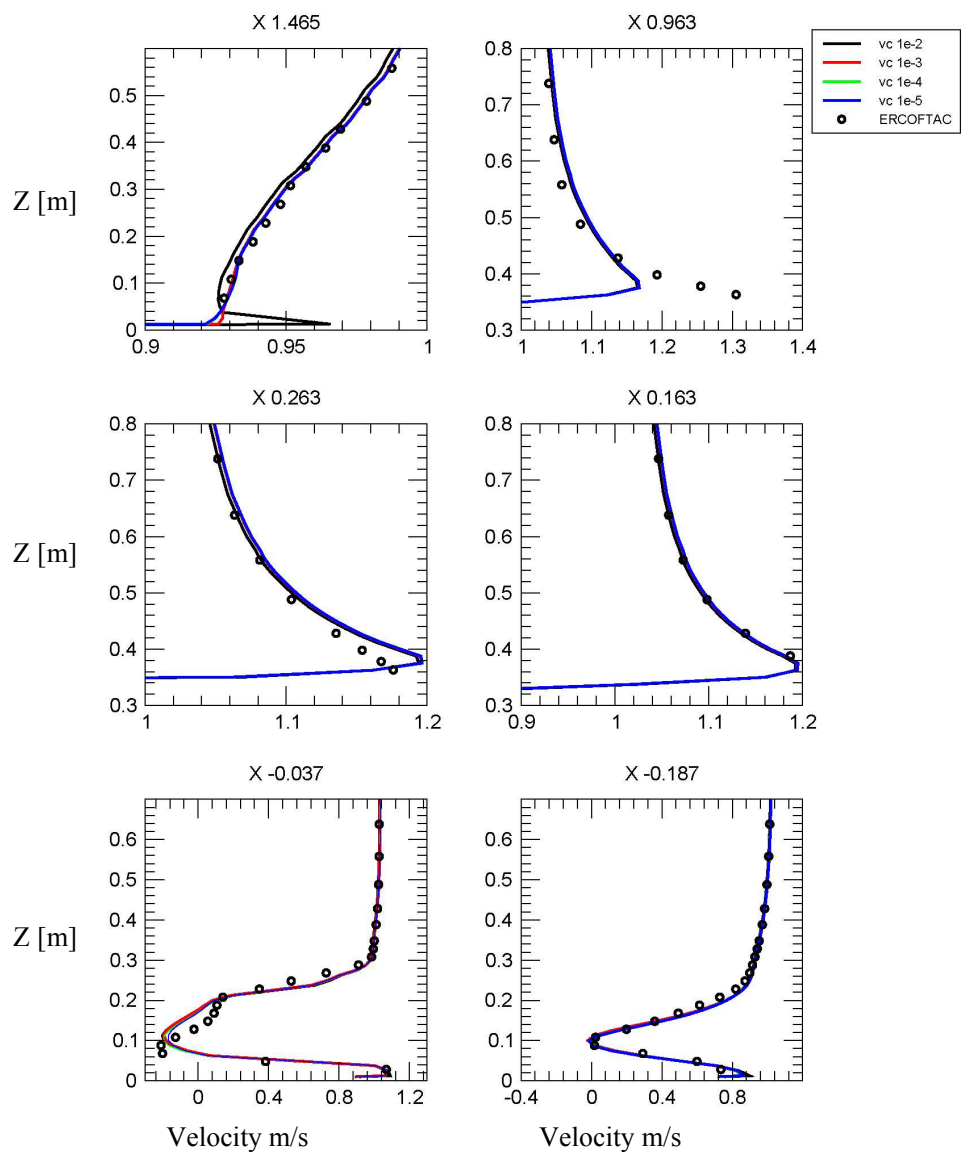

Figure 5: $\quad$ Velocity profiles on fine mesh, $25^{\circ}$ slant angle, $\mathrm{Co}_{\max }=8$. 
the wake region for locations $\mathrm{X}=-0.037$ and -0.187 , where velocities are over predicted. Generally, lowest difference can be encountered for very low and very high vorticity parameter $\varepsilon$, namely $\varepsilon=10^{-5}$ and $10^{-2}$. The largest error is for $\varepsilon=0.01$.

\subsection{Ahmed body $-35^{\circ}$ slant angle}

The Ahmed body geometry with the stronger slant angle leads to stronger separation and mainly the wake behind the body. This type of flow is more realistic to the real car geometry and more challenging case to model. Series of $\varepsilon$ parameter values were tested as well as time steps and mesh coarseness as for previous geometry. Here the results of velocity profiles for $35^{\circ}$ slant angle are presented. For the nature of the flow with the separation at the rear of the bluffbody, the results are presented mainly concerning this region in a series of figures. Generally the behaviour of the VC model is the same as it is on the previous geometry with $25^{\circ}$ slant angle, meaning there are not large differences in velocity profiles with respect to the changing $\varepsilon$ parameter, as it could have been seen in Figure 6 . The profiles for fine mesh are also shown in Figure 7 where very good fit could be seen for most of the profiles.

\subsection{Comparison of $\mathrm{VC}$ and RANS simulations}

Individual results of velocity profiles calculated with RANS models are not presented here, only comparison with VC. However, we can conclude that the velocity profiles at given cross sections show quite a good match for fine mesh in the wake region. In this case the resolution of the weak wake is sufficient. The problem is to capture the flow around the bluff body, where the mesh resolution is not capable of capturing the boundary layer. While the RANS on $25^{\circ}$ slant angle geometry is capable to capture the wake even on rather coarse mesh, it is not capable to do so on the $35^{\circ}$ geometry. Results obtained on fine mesh (the same as for VC calculations) are shown in Figures 8 and 9.

\section{Conclusions}

From all the computations we can conclude, that the VC method could be used for flows with small or no separation caused by adverse pressure gradient. It is also clear that the computational mesh for VC could be coarser compared to the RANS computations. Since the VC method is in principle transient, the resulting data must be averaged over reasonably long period of time. The method seems to be for the particular case of bluff body flow insensitive on the VC parameter $\varepsilon$ value, at least not that much as on the mesh. This conclusion, however, applies only to the particular case tested. In more complicated flow situation the effect of the VC parameter would be probably higher. The method must also be tested for grid independence as its success is dependent on the flow structure. From the 

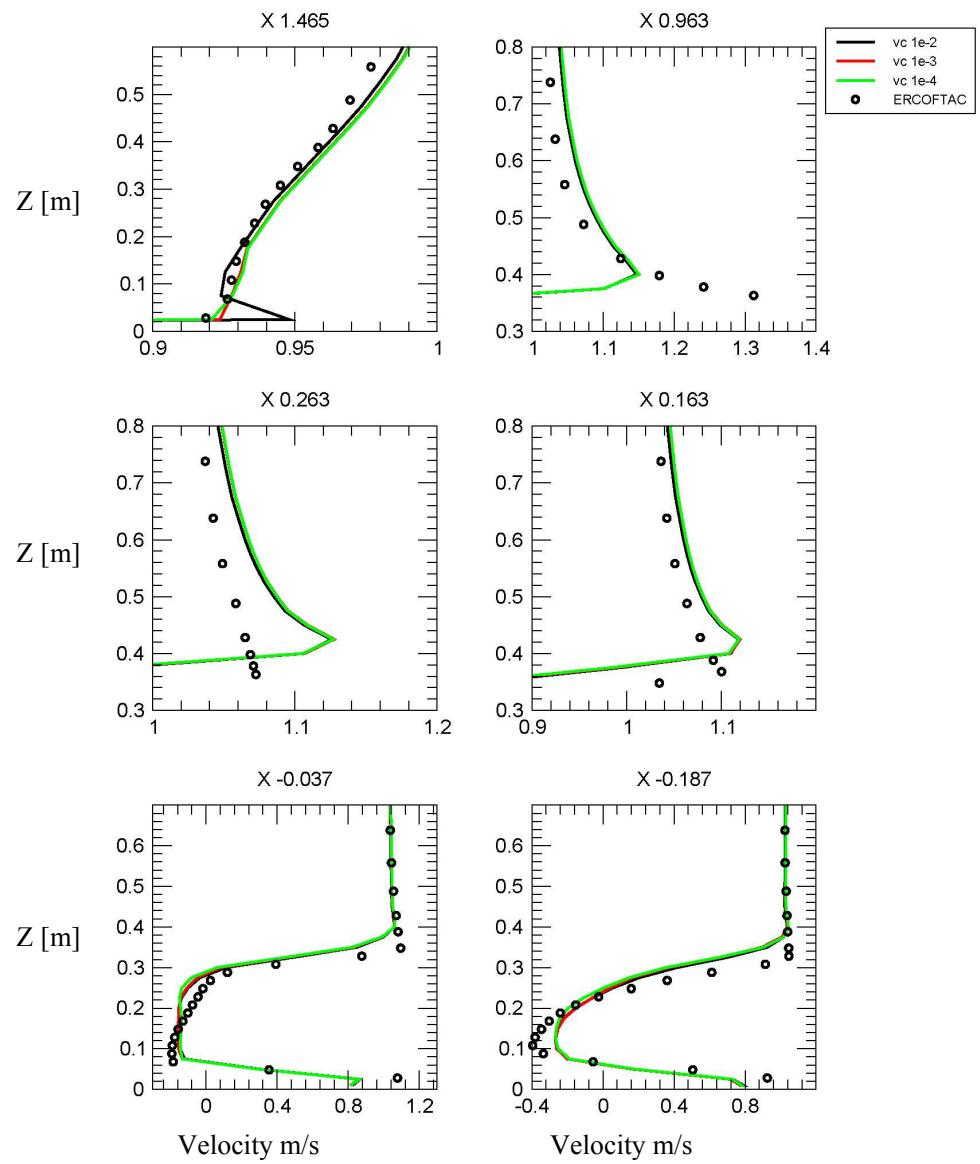

Figure 6: $\quad$ Velocity profiles on fine mesh, $35^{\circ}$ slant angle, $\mathrm{Co}_{\max }=8$.

Ahmed Body $35^{\circ}$ Slant: Symmetry Plane

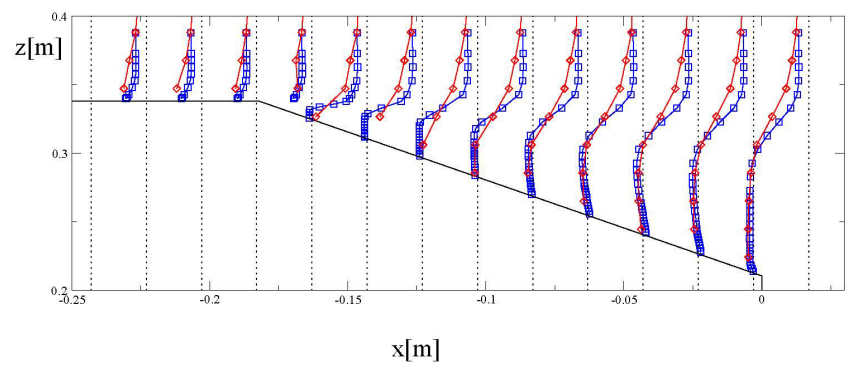

Figure 7: Velocity profiles on fine mesh; $\varepsilon=1 \times 10^{-3} ; \mathrm{Co}=10$. 

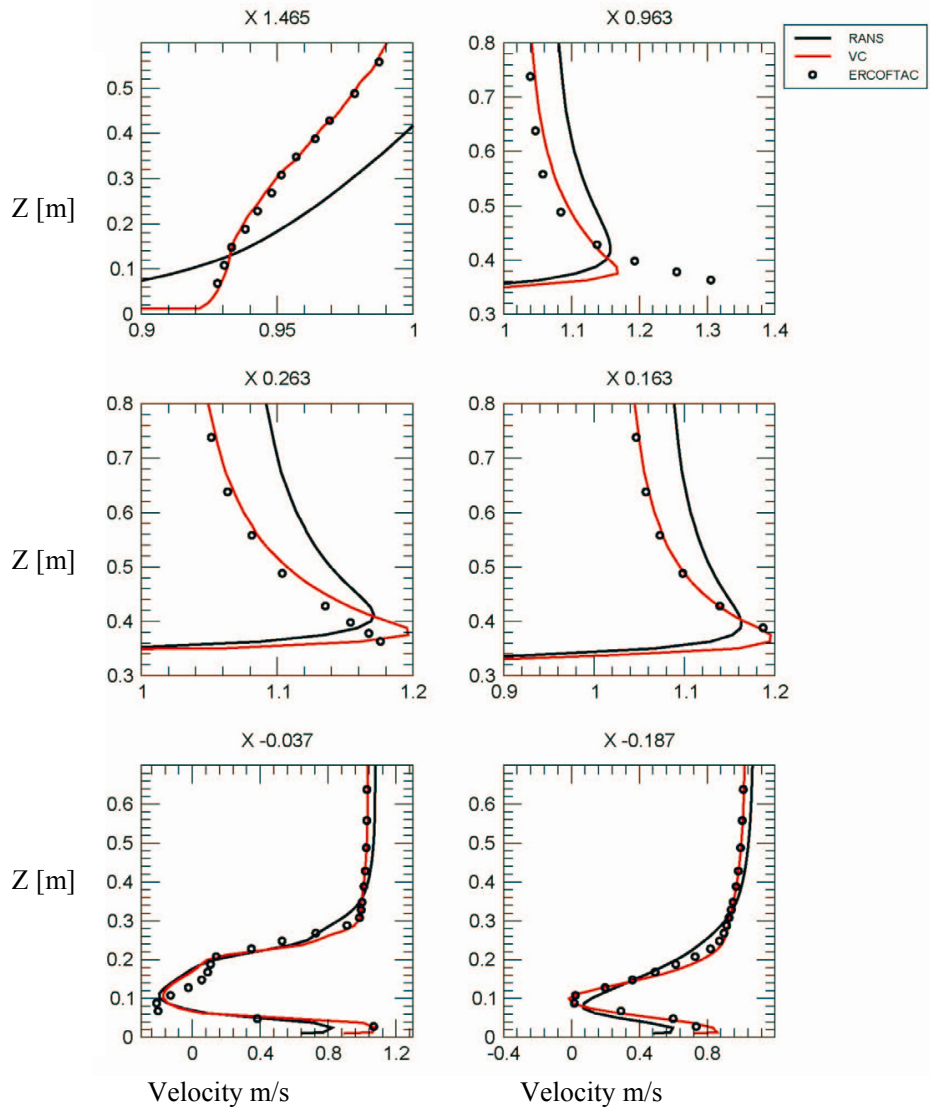

Figure 8: $\quad$ Velocity profiles; RANS and $\mathrm{VC}, 25^{\circ}$ slant angle.

point of view of practical computation the VC method brings improvement. Mainly in cases featuring transient behaviour, the speed-up is significant over RANS methods. Still the computation of Ahmed body flow with fine mesh would last several hours on multiple CPUs. In language of numbers, the RANS computations took two times more time than the VC computations on the same hardware setup. The VC main speed-up effect could lie in much more complicated flows where RANS or URANS simulation would demand grid of several tens of millions. The VC method could lead to faster prediction of turbulent flow, but its potential may lie in more complicated geometries, hence more complicated flow and turbulence structure configurations. 

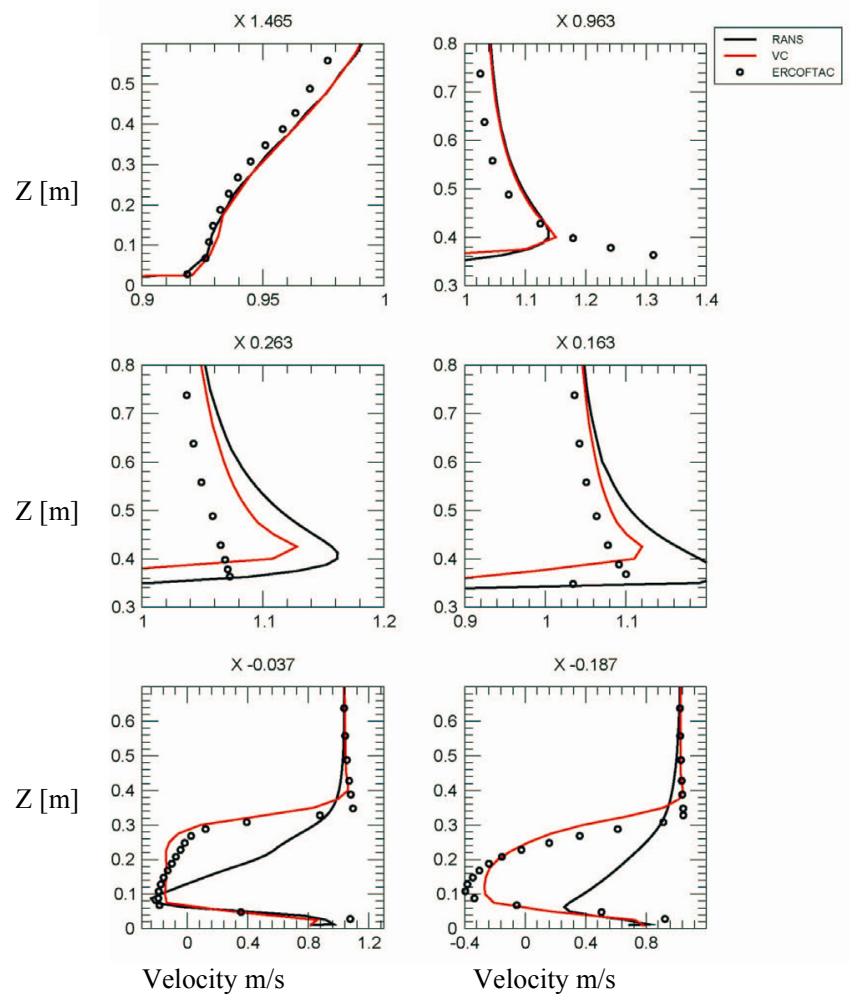

Figure 9: $\quad$ Velocity profiles; RANS and $\mathrm{VC}, 35^{\circ}$ slant angle.

\section{Acknowledgement}

The work was performed under the support from the project GA 101/08/0096 of the Czech Grant Agency, which is gratefully acknowledged.

\section{References}

[1] Fan, M., Wenren, Y., Dietz, W., Xiao, M., and Steinhoff, J.: “Computing blunt body flows on coarse grids using vorticity confinement", J. Fluids Engineering, 124(4):1-18, Dec 2002.

[2] Steinhoff, J., Lynn, N., and Wenren, Y.: Large Eddy Simulation: Computing Turbulent Flow Dynamics, Chapter 12: Turbulent Flow Simulations Using Vorticity Confinement: Cambridge University Press, 2006.

[3] Ahmed, S.R., Ramm, G.: Some salient features of the time-averaged ground vehicle wake, SAE Technical Paper 840300, 1984. 
[4] Lienhart H., Stoots C., Becker S., Flow and Turbulence Structures in the Wake of a Simplified Car Model (Ahmed Model), Measurements MOVAproject, online database: http://cfd.mace.manchester.ac.uk/ercoftac/.

[5] C. Benoit, Numerical simulation of 2D blade vortex interaction usány moving overset grids, in: 25th European Rotorcraft Forum, Roma, Italy, September 1999.

[6] C. Benoit, G. Jeanfaivre, 3D Inviscid isolated rotor and fuselage calculations using chimera and automatic Cartesian partitioning methods, in: AHS Aeromechanics 2000, Atlanta, GA, November 2000.

[7] A. Dadone, G. Hu, B. Grossman, Towards a better understanding of vorticity confinement methods in compressible flow, in: AIAA 2001 CFD Conference, Anaheim, CA, June 2001.

[8] A. Ochi, T. Aoyama, S. Saito, E. Shima, E. Yamakawa, BVI noise predictions by moving overlapped grid method, in: AHS 55th Annual Forum, Montréal, Québec, May 1999.

[9] M.R. Visbal, D.V. Gaitonde, High-order accurate methods for unsteady vortical flows on curvilinear meshes, in: AIAA 36th Aerospace Sciences, Meeting and Exhibit, Reno, NV, January 1998.

[10] J. Steinhoff, D. Underhill, Modification of the Euler equations for "vorticity confinement": Application to the computation of interacting vortex rings, Phys. Fluids 6 (8) (1994).

[11] Steinhoff, J., Dietz, W., Haas, S., Xiao, M., Lynn, M, and Fan, M: "Simulating Small Scale Features in Fluid Dynamics and Acoustics as NonLinear Solitary Waves", In 41st AIAA Aerospace Sciences Meeting and Exhibit, 2003: AIAA-2003-0078.

[12] Vaughn, M. E. Jr.: "An application of vorticity confinement to missile aerodynamic design", In 24th Applied Aerodynamics Conference. AIAA, June 2006: AIAA 2006-3866. 